OPEN ACCESS

Edited by:

Afsar Raza Naqvi,

University of Illinois at Chicago,

United States

Reviewed by:

Salvador Nares,

University of Illinois at Chicago,

United States

Henry Fergus Duncan,

Trinity College Dublin, Ireland

Gustavo Mendonca,

University of Michigan, United States

*Correspondence:

Asma A. Khan

Khana2@uthscsa.edu

Specialty section:

This article was submitted to

Viral Immunology,

a section of the journal

Frontiers in Immunology

Received: 03 October 2019 Accepted: 21 November 2019 Published: 06 December 2019

Citation:

Pluta L, Yousefi B, Damania B and Khan AA (2019) Endosomal TLR-8 Senses microRNA-1294 Resulting in the Production of NFḱB Dependent Cytokines. Front. Immunol. 10:2860.

doi: 10.3389/fimmu.2019.02860

\section{Endosomal TLR-8 Senses microRNA-1294 Resulting in the Production of NFḱB Dependent Cytokines}

\author{
Linda Pluta ${ }^{1}$, Babak Yousefi ${ }^{2}$, Blossom Damania ${ }^{1}$ and Asma A. Khan ${ }^{3 *}$ \\ ${ }^{1}$ Department of Microbiology and Immunology, School of Medicine, University of North Carolina at Chapel Hill, Chapel Hill, \\ NC, United States, ${ }^{2}$ Department of Oral and Craniofacial Health Sciences, School of Dentistry, University of North Carolina at \\ Chapel Hill, Chapel Hill, NC, United States, ${ }^{3}$ Department of Endodontics, Dental School, University of Texas Health Science \\ Center at San Antonio, San Antonio, TX, United States
}

The primary function of toll-like receptor 8 (TLR-8) is the detection of viruses and other microbial pathogens. Recent evidence suggests that TLR-8 also senses host microRNAs (miRNAs) and implicate TLR-8 in autoimmune disorders. This study examined the interaction between miR-1294 and TLR-8. We first performed a BLAST search to identify miRNAs with the same sequences as two core motifs of miR-1294. Next, we examined NFḰB activation induced by the binding of miR-1294 mimic to endosomal TLR-8. HEK-Blue ${ }^{\text {TM }}$ hTLR-8 cells (Invivogen), a HEK293 cell line co-transfected with human TLR-8 gene, were incubated with miR-1294 mimic. A TLR-8 agonist ssRNA40, was used as a positive control. Using the same experimental set up, we also examined the effects of miR-1294 and its two core motifs (Integrated DNA Technologies) on IL-8, $\mathrm{IL}-1 \beta$, and TNF $\alpha$. Data were analyzed using $t$-test or one-way ANOVA and Dunnets post-hoc test. Using miRCarta we identified 29 other mature human miRNAs or their precursors which contain the same core motifs as miR-1294. Our data show that miR1294 activates NFḰB in cells expressing TLR-8 $(\rho<0.05)$. miR-1294, and its core motifs induce expression of $I L-8, I L-1 \beta$, and TNF $\alpha$ via TLR8 activation $(p<0.05)$. This constitutes a novel mechanism by which endosomal TLR-8 senses host miRNAs resulting in the release of pro-inflammatory cytokines and thus potentially contributing to autoimmune disorders.

Keywords: microRNA, IL-8, cytokines, TLR-8, miR-1294, NF-кb

\section{INTRODUCTION}

Pathogen-associated molecular pattern receptors, including toll-like receptors (TLRs), are key molecules in the response to pathological stimuli such as bacterial endotoxins. TLRs are ubiquitously expressed by immune and non-immune cells including neurons, fibroblasts, endothelial cells, epithelial cells, and adipocytes. They recognize viral and microbial structures, and some endogenous molecules such as single stranded RNAs (1-3). Classic activation of TLRs depends upon the pathogen and the TLR expressing cell subtype (4).

To date, 11 human TLRs have been identified (5-8). Some TLRs, specifically TLRs 7, 8, and 9, recognize self molecules such as single stranded RNAs and play a role in autoimmunity. Recent studies show that TLR-7 recognizes microRNAs (miRNAs) resulting in the sensations of pain and itch (9). TLR-8 also recognizes miRNAs but differs from TLR-7 in multiple ways. TLR-8 is primarily 
expressed in monocytes, myeloid dendritic cells, and neutrophils while TLR-7 is expressed in B cells and plasmacytoid dendritic cells (10-12). TLR-8 is preferentially activated by ssRNA rich in AU sequences while TLR-7 is activated by sequences rich in GU (11). Moreover, unlike TLR-7, TLR-8 senses ssRNA through its ability to form secondary structures (13).

Nucleic acid-sensing TLRs (namely TLRs-7 and -8) are localized to endolysosomal compartments, where they encounter phagocytosed or endocytosed cargo (14). The subcellular localization of TLRs strategically favors the recognition of microbial (and not host) nucleic acids. Under normal conditions host nucleic acids are confined to other subcellular compartments and are not accessible to endosomal TLRs. Host nucleic acids in extracellular spaces are not taken up by endocytosis or phagocytosis. Instead they are rapidly degraded by nucleases unless they are stabilized by nucleic acid-binding proteins or are enclosed within extracellular vesicles $(15,16)$. In such conditions the host nucleic acids can reach endolysosomal compartments potentially leading to their recognition by TLRs and the development of autoimmunity.

The role of TLR- 8 in autoimmune disease is yet to be fully explored. TLR- 8 is implicated in the pathogenesis of several disorders including oral lichenoid reactions, irritable bowel syndrome (IBS), rheumatoid arthritis, systemic sclerosis and asthma (17-19). Blood levels of TLR-8 mRNA are higher in patients with systemic onset juvenile arthritis or Stills disease as compared to healthy controls (20). Furthermore, the levels of TLR- 8 mRNA in these patients correlates positively with levels of IL-1 $\beta$ mRNA. Transgenic mice expressing high levels of human TLR- 8 spontaneously develop arthritis while mice expressing low levels of TLR-8 do not (20). An ex vivo study incubated blood cells isolated from IBS patients with TLR agonists and assayed the supernatants for cytokine release (21). Cells from IBS patients responded to TLR-8 agonists in an exacerbated manner as compared to those from healthy controls. In a phase II clinical trial, administration of resiquimod, a TLR7/8 agonist, induced adverse events consistent with systemic cytokine induction at high doses (22). Studies on cancer show that tumor secreted miRNAs act as paracrine agonists of TLR-8 resulting in NFḱB activation and the release of pro-inflammatory cytokines (23). Thus, TLR- 8 activation by host miRs may induce the release of proinflammatory cytokines.

We conducted a series of clinical studies on TLRs and miRNA expression profiles. Our studies on biopsies of inflamed human dental pulps show that on comparing pulps from patients experiencing moderate to severe tooth pain to those with no pain or mild pain, TLR- 8 was the only differentially expressed TLR (24). In a separate study, we examined miRNA profiles in the blood from chronic pain patients. Specifically, these were patients with a single chronic pain condition-Vulvar Vestibulodynia alone (VBD alone) or patients with VBD and Irritable Bowel Syndrome (VBD + IBS) and compared them to pain-free controls (25). The miRNA profiles in the blood of chronic pain patients (VBD alone or VBD + IBS) differed from that of pain-free controls. We also noted an overlap in miRNA profiles between VBD alone and VBD + IBS. miR-1294 was differentially expressed in patients with VBD alone as well as in those with VBD + IBS. Another study reported results similar to our data with miR-1294, being differentially expressed in the blood of patients with chronic regional pain syndrome (26). To our knowledge, these are the only studies on miR-1294 in pain. Most studies on this miR are from the field of cancer research where it is reported to effect tumor progression (27-30).

Taken together these studies show that TLR- 8 and miRNAs (including miR-1294) are differentially expressed in pain patients. The current study examined whether TLR- 8 is activated by miR1294 and its core motifs resulting in the production of proinflammatory cytokines.

\section{MATERIALS AND METHODS}

\section{Cell Culture}

HEK-Blue ${ }^{\text {TM }}$ hTLR-8 cells (Invivogen, San Diego, CA) are a stable commercial cell line that co-expresses TLR8 gene and an optimized secreted embryonic alkaline phosphatase (SEAP) reporter gene into HEK293 cells. This SEAP reporter gene is controlled by IFN- $\beta$ promotor fused to five NF- $\kappa \mathrm{B}$ and AP-1 binding sites. With TLR8 ligand stimulation, $\mathrm{NF}-\kappa \mathrm{B}$ and $\mathrm{AP}-1$ are induced to produce SEAP.

Cells were grown according to manufactures' instruction in a maintenance media of Dulbecco's modified Eagle Media plus high glucose $(4.5 \mathrm{~g} / \mathrm{l})$ and L-Glutamine $(4 \mathrm{mM})$ (HyClone-GE Health Sciences, Logan, UT) supplemented with 10\% v/v Fetal Bovine Serum (Gibco, Gaithersburg, MD), $50 \mathrm{U} / \mathrm{ml}$ Penicillin (Gibco), $50 \mu \mathrm{g} / \mathrm{ml}$ Streptomycin (Gibco), $100 \mu \mathrm{g} / \mathrm{ml}$ Normocin (Invivogen), $30 \mu \mathrm{g} / \mathrm{ml}$ Blasticidin (Invivogen), and $100 \mu \mathrm{g} / \mathrm{ml}$ Zeocin (Invivogen) under standard conditions of $37^{\circ} \mathrm{C}$, $5 \% \mathrm{CO}_{2}$.

\section{NF-кB Activation}

LyoVec complexed with miR-1294 was added to the HEKBlue $^{\text {TM }}$ hTLR-8 cells. We initially used three different concentrations of miR1294 complexes- 200, 400, and $600 \mathrm{nM}$ and incubated the cells for 18 and $24 \mathrm{~h}$. As we got a robust response at $400 \mathrm{nM}$ and $24 \mathrm{~h}$, all subsequent experiments were conducted at that concentration and time point. Positive controls for the experiment were R848 (Resiquimod: an Imidazoquinoline compound, Invivogen), ssRNA40 (mimics viral ssRNA, Invivogen), and LyoVec transfection control (Invivogen). Negative controls included ssRNA41 (derived from ssRNA40 with replacement of U's by A's), LyoVec, hTLR- 8 cells only and the parental line of hTLR- 8 cells. Three biological replicate experiments plated on separate days were performed with individual samples placed in triplicate on each plate. Plates were analyzed spectrophotometrically at $620 \mathrm{~nm}$ wavelength on Spectra Max M2 (Molecular Devices, Sunnyvale, CA).

\section{Cytokine Gene and Protein Expression}

We used the same experimental setup as described above to examine expression of cytokines at the transcript and protein levels. In addition to miR-1294 mimic, we also 
challenged the cells with two of its core motifs- Motif AAUUGUUG and Motif B- AUUGUUA. To facilitate efficient gene expression analysis, we utilized the TaqMan ${ }^{\circledR}$ Gene Expression Cells-to-CT ${ }^{\mathrm{TM}}$ Kit (Applied Biosystems/Thermo, Foster City, CA). Taqman primer assays were from Quant Studio 6 (Applied Biosystems/Thermo).

For analysis of protein expression, supernatants were collected from the plates after $24 \mathrm{~h}$ of incubation and placed on ice. Each sample was centrifuged (1,400 rpm for $1 \mathrm{~min})$ to remove cellular debris and the supernatants placed in $1.5 \mathrm{ml}$ micro-centrifuge tubes. Human Luminex ( $\&$ \&D systems, Minneapolis, MN) bead-based multiplex assays were used for the quantitation of the proteins. Multiplex assays were performed according to reagent manufacturer's instructions using a Bio-Plex 200 system (Bio-Rad, Hercules, CA) with Bio-Plex Manager v. 6.0 operating software. Samples along with the standards were performed in duplicate on multiple 96 well-format plates. All experiments were repeated at least three times.

TABLE 1 | In silico analysis identifying miRs that contain the core AUUGUUG.

\begin{tabular}{llll}
\hline Name & Accession & miRCarta & Entity \\
\hline hsa-mir-181d & Ml0003139 & hsa-194-1489.1 & Precursor \\
hsa-miR-181d-5p & MIMAT0002821 & m-194 & miRNA \\
hsa-mir-3065 & Ml0014228 & hsa-454-247.1 & Precursor \\
hsa-miR-3065-3p & MIMAT0015378 & m-247 & miRNA \\
hsa-mir-3145 & Ml0014170 & hsa-1645-1465.1 & Precursor \\
hsa-mir-3613 & Ml0016003 & hsa-259-525.1 & Precursor \\
hsa-mir-3655 & MI0016055 & hsa-2207.1 & Precursor \\
hsa-mir-4774 & MI0017417 & hsa-2068-2326.1 & Precursor \\
hsa-miR-601 & MIMAT0003269 & m-2255 & miRNA \\
hsa-mir-601 & MI0003614 & hsa-2255.1 & Precursor \\
hsa-mir-633 & MI0003648 & hsa-2111.1 & Precursor \\
hsa-mir-6502 & MI0022214 & hsa-810-2463.1 & precursor \\
hsa-miR-6502-5p & MIMAT0025460 & m-810 & miRNA \\
hsa-mir-6759 & MI0022604 & hsa-2074-2271.1 & Precursor \\
hsa-mir-7849 & MI0025519 & hsa-1426.1 & Precursor \\
hsa-miR-7849-3p & MIMAT0030424 & m-1426 & miRNA \\
hsa-mir-8066 & MI0025902 & hsa-2376.1 & Precursor \\
hsa-mir-98 & MI0000100 & hsa-143-367.1 & Precursor \\
& & &
\end{tabular}

TABLE 2 | In silico analysis identifying miRs that contain the core AUUGUUA.

\begin{tabular}{llll}
\hline Name & Accession & miRCarta & Entity \\
\hline hsa-mir-132 & MI0000449 & hsa-414-138.1 & Precursor \\
hsa-miR-132-5p & MIMAT0004594 & m-414 & miRNA \\
hsa-miR-449a & MIMAT0001541 & m-696 & miRNA \\
hsa-mir-449a & MI0001648 & hsa-696.1 & precursor \\
hsa-mir-449b & MI0003673 & hsa-923-1052.1 & Precursor \\
hsa-miR-449b-5p & MIMAT0003327 & m-923 & miRNA \\
hsa-mir-569 & MI0003576 & hsa-2357.1 & Precursor
\end{tabular}

\section{Identification of miRNAs With Similar \\ Cores}

We used the Basic Local alignment Search Tool (BLAST) to identify other microRNA with sequences similar to the core motifs AUUGUUG and AUUGUUA used in this study. The search was conducted on miRCarta, a central repository for miRNA candidates, and was restricted to human microRNAs.

\section{Statistical Analysis}

Data were analyzed using GraphPad Prism software (La Jolla, CA, USA). Data is shown as mean \pm SEM. Data were analyzed by

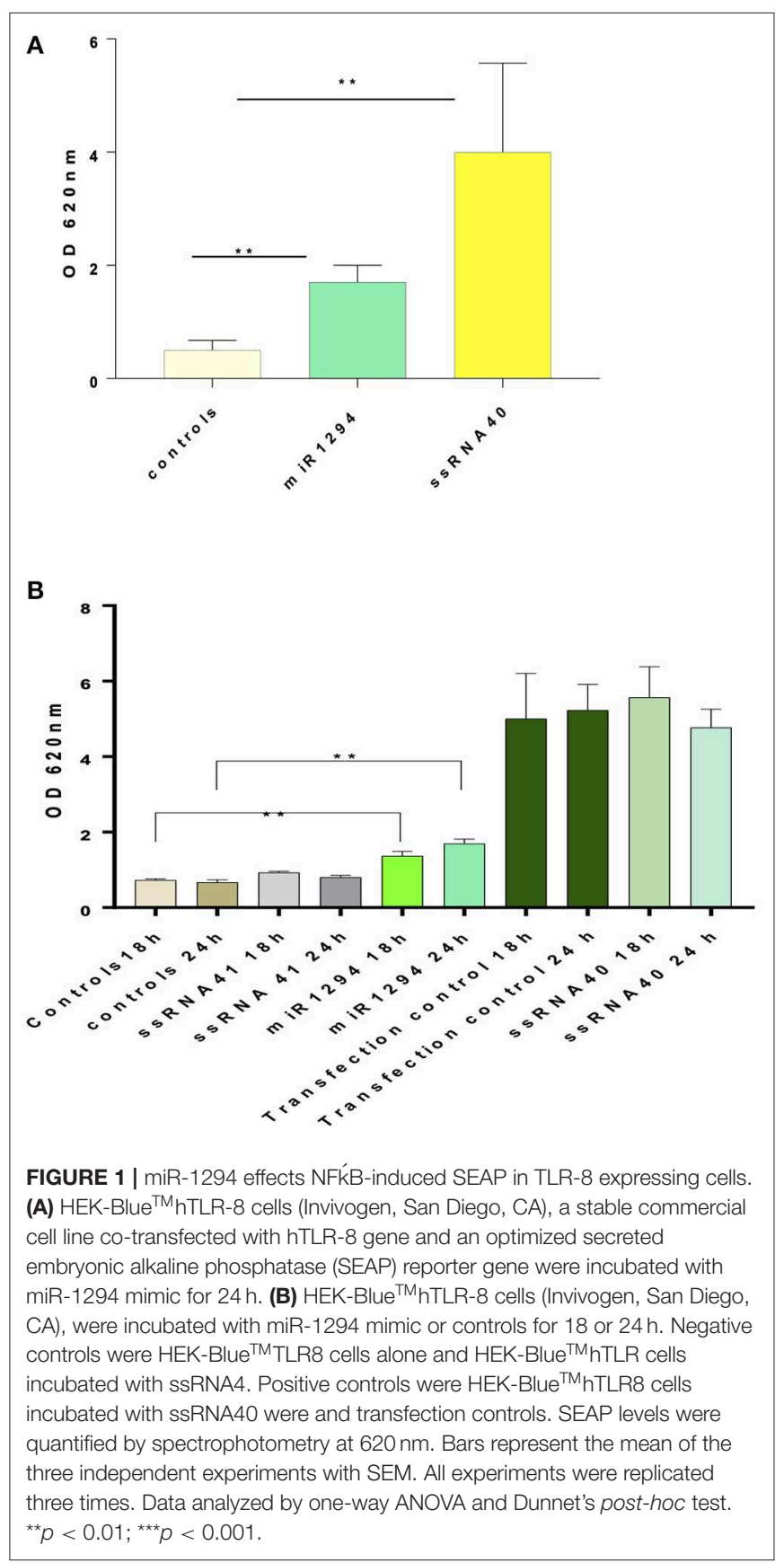


one-way ANOVA and Dunnets post-hoc test. $P$-values $<0.05$ were considered significant.

\section{RESULTS}

\section{Several microRNAs Express the Same Core Motifs as miR-1294}

In silico analysis identified 18 mature or precursor miRNAs which also contain the core motif AUUGUUG (Table 1) and seven mature or precursor miRNAs which contain the motif AUUGUUA (Table 2). Some of these have been reported to be expressed at higher levels in diseased tissue biopsies or in extracellular vesicles from patient populations. For example, miRNA-449a is expressed in high levels in breast cancer (31). Extracellular vesicles secreted by human carcinoma cell lines contain miR181d (32) and extracellular vesicles from patients with chronic regional pain syndrome contain $>4$-folds higher levels of miR-98 as compared to healthy controls (26). Deep sequencing of extracellular vesicles released by colon cancer cell lines shows that a subpopulation of them are enriched with both the miR-181 family as well as miR-98 (32). Given that extracellular vesicles are a form of intercellular communication, it is likely that the identified miRNAs can reach and activate endosomal TLR-8 to induce inflammation in target cells distant from the site of origin. Prior studies report that extracellular vesicular miRNAs released from cancer cells activate TLRs in immune cells (23). The target cells may also include neurons in the central nervous system as miRNAs packaged in extracellular vesicles are known to impact neuronal function. The functions of extracellular vesicular miRNAs are yet unclear. Further research is needed to understand how they modulate their target cells.

\section{miR-1294 Induces NFKB Activation via TLR-8 Binding}

Incubation of HEK-Blue ${ }^{\text {TM }}$ hTLR-8 cells with miR-1294 induced robust production of NFKB-inducible secreted
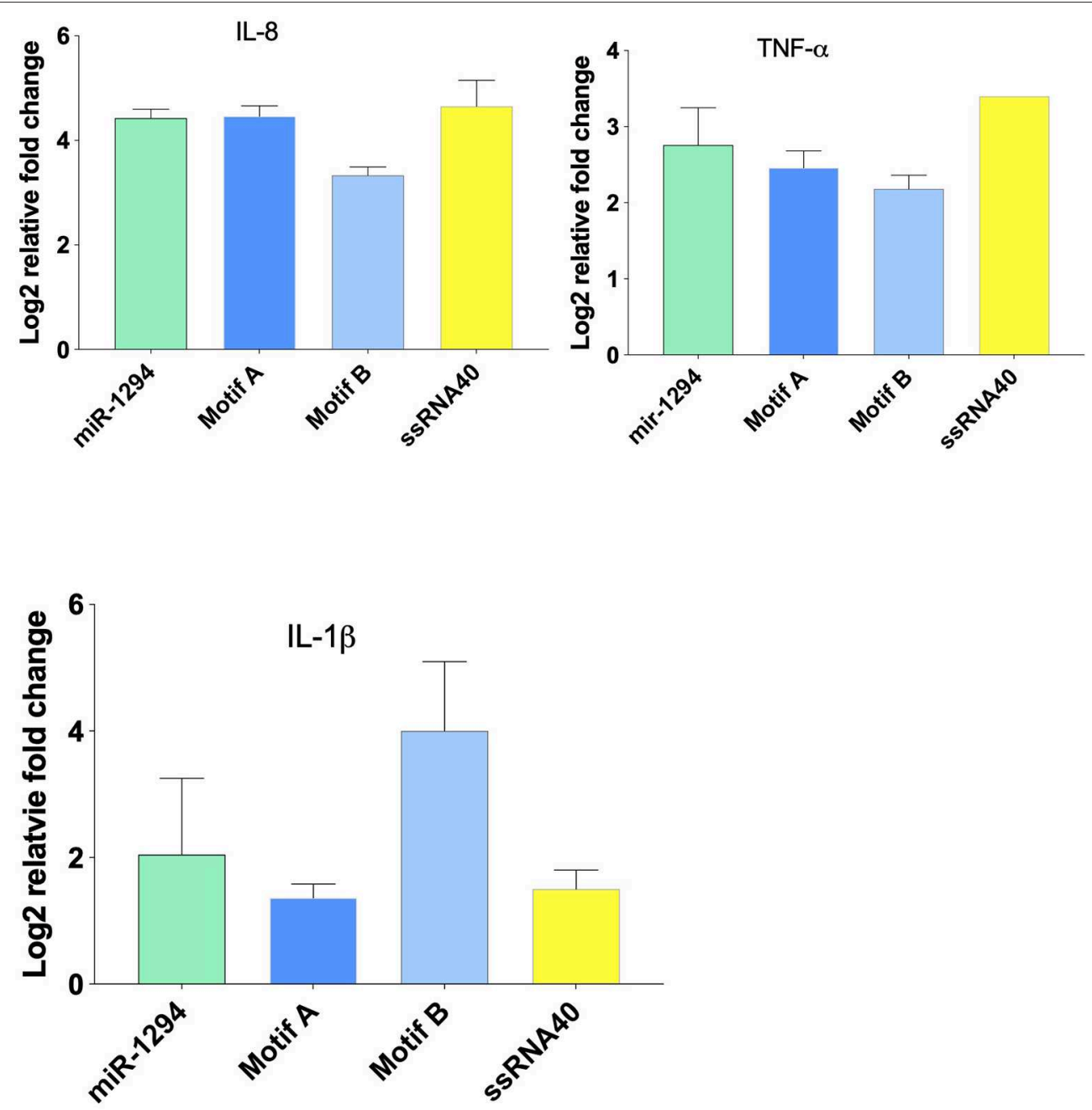

FIGURE 2 | miR-1294 and its core motifs induce expression of IL-8, IL-1 $\beta$, and TNF $\alpha$ mRNA. HEK-Blue ${ }^{\text {TM }}$ hTLR8 cells (Invivogen, San Diego, CA), were incubated with miR-1294 mimic and its two core motifs (Motif A-AUUGUUG and Motif B- AUUGUUA) for 24h. ssRNA40, a TLR8 agonist, was used as a control. All experiments were replicated three times. 
embryonic alkaline phosphatase (SEAP) $(p<0.01)$ (Figure 1A). Incubation with ssRNA40, a known TLR-8 agonist also induced SEAP production while ssRNA41 did not (Figure 1B). ssRNA41 is derived from ssRNA40 by replacement of all $U$ nucleotides with adenosine and was used as a negative control.

\section{miR-1294 and Its Core Motifs Induce Cytokine Expression}

Incubation of HEK-Blue ${ }^{\mathrm{TM}}$ hTLR-8 cells with miR-1294 and its two core motifs induced significant increase in expression of IL8 , TNF $\alpha$, and IL-1 $\beta$ mRNA (Figure 2 ). The effects of the two core motifs on IL- 8 were not identical. Motif A induced a more robust expression of IL- 8 mRNA and protein than motif $\mathrm{B}$. This raises the possibility that the downstream signaling induced by these motifs differs.

On examining cytokine protein expression in the supernatants, it was noted that miR-1294 and/or both of its core motifs induced significant increase in IL-8 and TNF $\alpha$ but not IL-1 $\beta$ ( $p<0.05$; Figure 3). The dissociation between IL-1 $\beta$ mRNA and protein synthesis has been reported in prior studies (33-35). The kinetics of IL-1 secretion are unique as compared to other secreted proteins. Large pools of precursor IL-1 accumulate intracellularly prior to release (35). Given that we assayed the supernatants, and not the cells, for IL- $1 \beta$ levels, it is likely that the cells themselves contained high levels of IL-1 $\beta$ or its precursor.

\section{DISCUSSION}

Altered expression of microRNAs is noted in several disorders including systemic sclerosis, inflammatory bowel disease, psoriatic arthritis, spinal muscular atrophy, and rheumatoid arthritis $(21,36,37)$. miRNA expression profiles have been reported in effector cells as well as body fluids such as plasma and serum. In addition, altered miRNA expression has been documented in cancer, autoimmune disease, neurological disease, and cardiovascular disease. The distinct expression profiles of miRNAs in disease have raised the possibility that miRNA profiles may be used as biomarkers. However, the functional role of these miRNAs is only just being explored. Here we provide evidence that miR-1294 and its core motifs

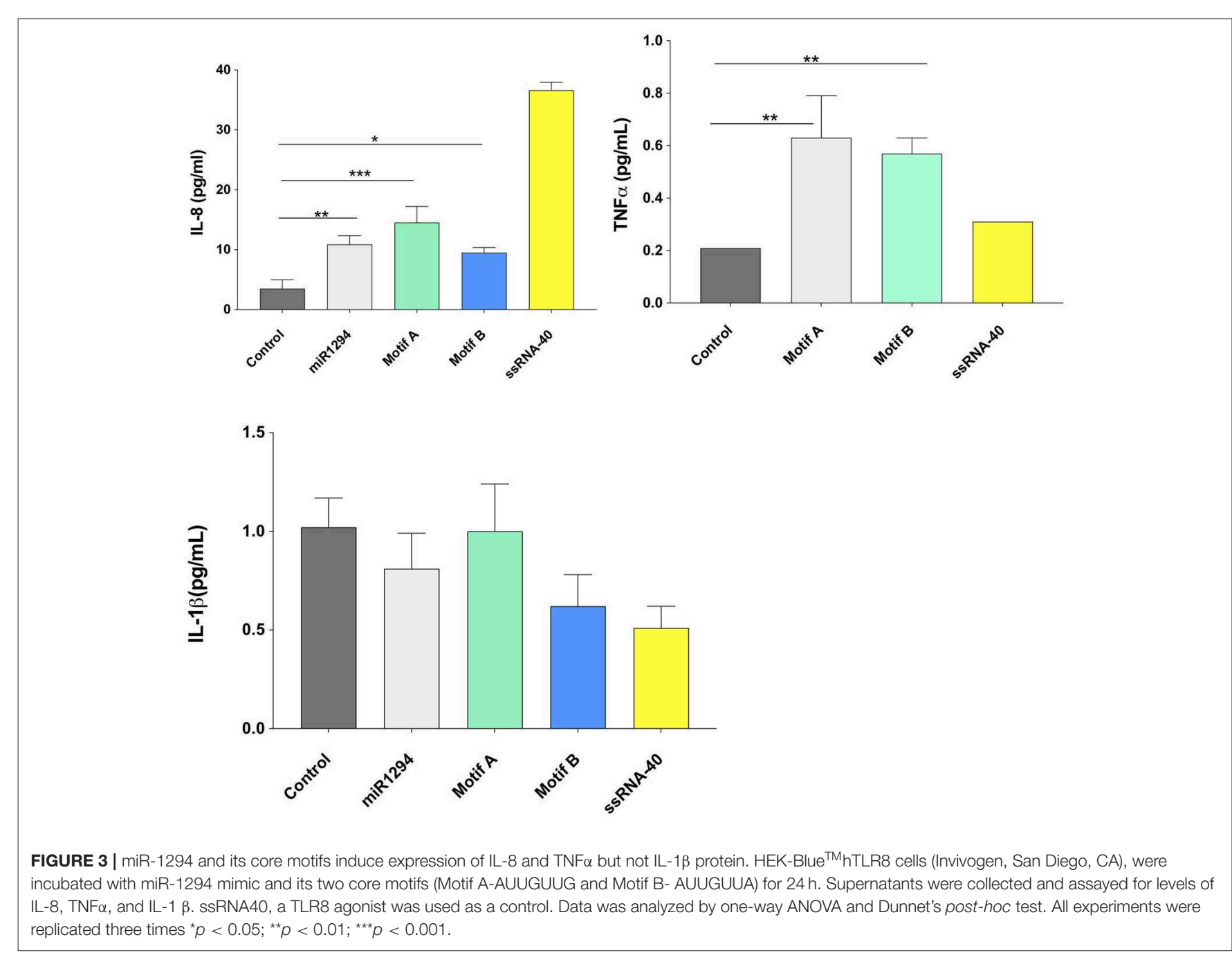


bind TLR-8 resulting in NFḱB activation and induction of pro-inflammatory cytokines.

Our initial studies on $\mathrm{miR}$ and TLR expression were conducted using biopsies of inflamed pulp tissues. Pulpitis represents an immune response to bacterial infection (3840). Infections of the dental pulp typically involve a mixed, predominantly gram-negative and anaerobic bacterial flora and contain high levels of TNF $\alpha$, IL-1, and IL-8 (41-44). Pulpal fibroblasts, immune cells, and neurons express TLRs, including TLR8, which recognize microbial components and activate $\mathrm{NF \kappa B}$, which in turn activates pro-inflammatory genes $(40,44)$. Pulpitis can be symptomatic with patients experiencing moderate to severe pain or it can be asymptomatic with no clinical symptoms of pain.

Using in silico analysis we identified several other miRNAs and their precursors which have the core motifs AUUGUUA and AUUGUUG. This raises the possibility that these miRNAs can bind and activate TLR-8 and induce cytokine synthesis. Some of the miRNAs that were identified are expressed at high levels in tissue biopsies and in exosomes. For example, miRNA$449 \mathrm{a}$ is expressed in high levels in breast cancer (31). Exosomes secreted by human carcinoma cell lines contain miR181d (32). Exosomes from patients with chronic regional pain syndrome contain $>4$-folds higher levels of miR-98 as compared to healthy controls (26). Given that exosomes are a form of intercellular communication, it is likely that the identified miRNAs can induce inflammation in target cells distant from the site of origin. Prior studies report that exosomal miRNAs released from cancer cells activate TLRs in immune cells (23). The target cells may also include neurons in the central nervous system as miRNAs packaged in exosomes are known to impact neuronal function. The function of exosomal miRNAs are yet unclear. Further research is needed to understand how they modulate their target cells.

IL-8 is a functionally diverse cytokine. It plays an important role in the immune response by inducing migratory and phagocytic activity and promoting angiogenesis. It is secreted by numerous cell types including endothelial cells, monocytes, neutrophils, and others (6). The synthesis and release of IL-8 is

\section{REFERENCES}

1. Lien E, Sellati TJ, Yoshimura A, Flo TH, Rawadi G, Finberg RW, et al. Tolllike receptor 2 functions as a pattern recognition receptor for diverse bacterial products. J Biol Chem. (1999) 274:33419-25. doi: 10.1074/jbc.274.47.33419

2. Wadachi R, Hargreaves KM. Trigeminal nociceptors express TLR-4 and CD14, a mechanism for pain due to infection. J Dental Res. (2006) 85:4953. doi: 10.1177/154405910608500108

3. Friedman JM, Jones PA. MicroRNAs: critical mediators of differentiation, development and disease. Swiss Med Wkly. (2009) 139:466-72.

4. Akira $S$, Uematsu $S$, Takeuchi O. Pathogen recognition and innate immunity. Cell. (2006) 124:783-801. doi: 10.1016/j.cell.2006.02.015

5. Olivieri F, Rippo MR, Prattichizzo F, Babini L, Graciotti L, Recchioni R, et al. Toll like receptor signaling in "inflammaging": microRNA as new players. Immun Ageing. (2013) 10:11. doi: 10.1186/1742-4933-10-11

6. Matsushima N, Miyashita H, Enkhbayar P, Kretsinger RH. Comparative geometrical analysis of leucine-rich repeat structures in the nod-like and tolllike receptors in vertebrate innate immunity. Biomolecules. (2015) 5:195578. doi: 10.3390/biom5031955 induced by various triggers including viral infections, bacterial lipopolysaccharides and cytokines such as IL-1, TNF $\alpha$, Il-17, etc. Additionally, it can also inhibit the antiviral effects of interferon$\alpha$ and thus enhance viral dissemination (45). Multiple lines of evidence support the role of IL-8 in pain hypersensitivity (46, 47). High levels of IL-8 are noted in diseases including chronic regional pain syndrome and pulpitis, both of which are associated with reports of intense pain $(48,49)$. To our knowledge this is the first report on activation of TLR- 8 by host microRNA resulting in increased IL-8 expression.

In conclusion, our data show that miR-1294 and its two core motifs activate NFKB via TLR-8 resulting in increased expression of the cytokines IL- 8 , TNF $\alpha$, and IL- $1 \beta$. The core motifs examined here are also contained in other microRNAs. Thus, our findings have broader implications beyond miR1294 and suggest that multiple miRNAs can potentially activate TLR-8 and induce cytokine synthesis. Given that aberrant $\mathrm{miR}$ expression is noted in multiple diseases, future research should be directed toward miR-TLR-8 interactions in various disease models.

\section{DATA AVAILABILITY STATEMENT}

The datasets generated for this study are available on request to the corresponding author.

\section{AUTHOR CONTRIBUTIONS}

AK contributed toward hypothesis generation, experiment design, data interpretation, and manuscript preparation. LP and $\mathrm{BY}$ conducted the experiments. $\mathrm{BD}$ contributed toward data interpretation.

\section{FUNDING}

This work was supported by the National Institutes of Health, National Institute of Dental and Craniofacial Research (Grant number- R56 DE025399), and Short term Fellowship Award, UNC-Chapel Hill, Adams School of Dentistry.

7. Schurz H, Daya M, Moller M, Hoal EG, Salie M. TLR1, 2, 4, 6 and 9 variants associated with tuberculosis susceptibility: a systematic review and meta-analysis. PLoS ONE. (2015) 10:139711. doi: 10.1371/journal.pone.01 39711

8. Mitchell S, Vargas J, Hoffmann A. Signaling via the NFкB system. Wiley Interdiscip Rev Syst Biol Med. (2016) 8:227-41. doi: 10.1002/wsbm.1331

9. Park CK, Xu ZZ, Berta T, Han Q, Chen G, Liu XJ, et al. Extracellular microRNAs activate nociceptor neurons to elicit pain via TLR7 and TRPA1. Neuron. (2014) 82:47-54. doi: 10.1016/j.neuron.2014.02.011

10. Hattermann K, Picard S, Borgeat M, Leclerc P, Pouliot M, Borgeat P. The tolllike receptor 7/8-ligand resiquimod (R-848) primes human neutrophils for leukotriene B4, prostaglandin E2 and platelet-activating factor biosynthesis. FASEB J. (2007) 21:1575-85. doi: 10.1096/fj.06-7457com

11. Forsbach A, Nemorin JG, Montino C, Muller C, Samulowitz U, Vicari $\mathrm{AP}$, et al. Identification of RNA sequence motifs stimulating sequencespecific TLR8-dependent immune responses. J Immunol. (2008) 180:372938. doi: 10.4049/jimmunol.180.6.3729

12. Janke M, Poth J, Wimmenauer V, Giese T, Coch C, Barchet W, et al. Selective and direct activation of human neutrophils but not eosinophils 
by Toll-like receptor 8. J Allergy Clin Immunol. (2009) 123:102633. doi: $10.1016 /$ j.jaci.2009.02.015

13. Sarvestani ST, Williams BR, Gantier MP. Human Toll-like receptor 8 can be cool too: implications for foreign RNA sensing. J Interferon Cytokine Res. (2012) 32:350-61. doi: 10.1089/jir.2012.0014

14. Kawai T, Akira S. TLR and signaling. Cell Death Differ. (2006) 13:81625. doi: $10.1038 /$ sj.cdd. 4401850

15. Boon RA, Vickers KC. Intercellular transport of microRNAs. Arterioscler Thromb Vasc Biol. (2013) 33:186-92. doi: 10.1161/ATVBAHA.112.300139

16. Sun Z, Shi K, Yang S, Liu J, Zhou Q, Wang G, et al. Effect of exosomal miRNA on cancer biology and clinical applications. Mol Cancer. (2018) 17:147. doi: 10.1186/s12943-018-0897-7

17. Duffy L, OReilly SC. Toll-like receptors in the pathogenesis of autoimmune diseases: recent and emerging translational developments. Immunotargets Ther. (2016) 5:69-80. doi: 10.2147/ITT.S89795

18. Ah Kioon MD, Tripodo C, Fernandez D, Kirou KA, Spiera RF, Crow MK, et al. Plasmacytoid dendritic cells promote systemic sclerosis with a key role for TLR8. Sci Transl Med. (2018) 10:8458. doi: 10.1126/scitranslmed.aam8458

19. Salem A, Mustafa R, Listyarifah D, Al-Samadi A, Barreto G, Nordstrom $\mathrm{D}$, et al. Altered expression of toll-like receptors in human oral epithelium in oral lichenoid reactions. Am J Dermatopathol. (2017) 39:811-8. doi: 10.1097/DAD.0000000000000807

20. Guiducci C, Gong M, Cepika AM, Xu Z, Tripodo C, Bennett L, et al. RNA recognition by human TLR8 can lead to autoimmune inflammation. J Exp Med. (2013) 210:2903-19. doi: 10.1084/jem.20131044

21. McKernan DP, Gaszner G, Quigley EM, Cryan JF, Dinan TG. Altered peripheral toll-like receptor responses in the irritable bowel syndrome. Aliment Pharmacol Ther. (2011) 33:104552. doi: $10.1111 / j .1365-2036.2011 .04624 . x$

22. Pockros PJ, Guyader D, Patton H, Tong MJ, Wright T, McHutchison JG, et al. Oral resiquimod in chronic HCV infection: safety and efficacy in 2 placebo-controlled, double-blind phase IIa studies. J Hepatol. (2007) 47:17482. doi: 10.1016/j.jhep.2007.02.025

23. Fabbri M, Paone A, Calore F, Galli R, Gaudio E, Santhanam R, et al. MicroRNAs bind to Toll-like receptors to induce prometastatic inflammatory response. Proc Natl Acad Sci USA. (2012) 109:E211016. doi: 10.1073/pnas. 1209414109

24. Galicia JC, Henson BR, Parker JS, Khan AA. Gene expression profile of pulpitis. Genes Immun. (2016) 17:239-43. doi: 10.1038/gene.2016.14

25. Ciszek BP, Khan AA, Dang H, Slade GD, Smith S, Bair E, et al. MicroRNA expression profiles differentiate chronic pain condition subtypes. Transl Res. (2015) 166:706-20.e11. doi: 10.1016/j.trsl.2015.06.008

26. McDonald MK, Tian YZ, Qureshi RA, Gormley M, Ertel A, Gao R, et al. Functional significance of macrophage-derived exosomes in inflammation and pain. Pain. (2014) 155:1527-39. doi: 10.1016/j.pain.2014.04.029

27. Liu K, Li L, Rusidanmu A, Wang Y, Lv X. Down-regulation of MiR1294 is related to dismal prognosis of patients with esophageal squamous cell carcinoma through elevating C-MYC expression. Cell Physiol Biochem. (2015) 36:100-10. doi: 10.1159/000374056

28. Shi YX, Ye BL, Hu BR, Ruan XJ. Expression of miR-1294 is downregulated and predicts a poor prognosis in gastric cancer. Eur Rev Med Pharmacol Sci. (2018) 22:5525-5530. doi: 10.26355/eurrev_201809_15813

29. Wang Z, Yan J, Zou T, Gao H. MicroRNA-1294 inhibited oral squamous cell carcinoma growth by targeting c-Myc. Oncol Lett. (2018) 16:224350. doi: $10.3892 / \mathrm{ol} .2018 .8967$

30. Zhang Y, Huang S, Guo Y, Li L. MiR-1294 confers cisplatin resistance in ovarian cancer cells by targeting IGF1R. Biomed Pharmacother. (2018) 106:1357-63. doi: 10.1016/j.biopha.2018.07.059

31. Shi W, Bruce J, Lee M, Yue S, Rowe M, Pintilie M, et al. MiR-449a promotes breast cancer progression by targeting CRIP2. Oncotarget. (2016) 7:1890618. doi: $10.18632 /$ oncotarget. 7753

32. Ji H, Chen M, Greening DW, He W, Rai A, Zhang W, et al. Deep sequencing of RNA from three different extracellular vesicle (EV) subtypes released from the human LIM1863 colon cancer cell line uncovers distinct miRNA-enrichment signatures. PLoS ONE. (2014) 9:e110314. doi: 10.1371/journal.pone.0110314
33. Dinarello CA, Schindler R. Dissociation of transcription from translation of human IL-1-beta: the induction of steady state mRNA by adherence or recombinant C5a in the absence of translation. Prog Clin Biol Res. (1990) 349:195-204.

34. Schindler R, Clark BD, Dinarello CA. Dissociation between interleukin-1 beta mRNA and protein synthesis in human peripheral blood mononuclear cells. $J$ Biol Chem. (1990) 265:10232-7.

35. Hazuda DJ, Lee JC, Young PR. The kinetics of interleukin 1 secretion from activated monocytes. Differences between interleukin 1 alpha and interleukin 1 beta. J Biol Chem. (1988) 263:8473-9.

36. Li X, Kroin JS, Kc R, Gibson G, Chen D, Corbett GT, et al. Altered spinal microRNA-146a and the microRNA-183 cluster contribute to osteoarthritic pain in knee joints. J Bone Miner Res. (2013) 28:251222. doi: $10.1002 / \mathrm{jbmr} .2002$

37. Georgantas RW, Streicher K, Greenberg SA, Greenlees LM, Zhu W, Brohawn $\mathrm{PZ}$, et al. Inhibition of myogenic microRNAs 1, 133, and 206 by inflammatory cytokines links inflammation and muscle degeneration in adult inflammatory myopathies. Arthr Rheumatol. (2014) 66:1022-33. doi: 10.1002/art.38292

38. Kakehashi S, Stanley HR, Fitzgerald RJ. The effects of surgical exposures of dental pulps in germfree and conventional laboratory rats. J South Calif Dent Assoc. (1966) 34:449-51.

39. Warfvinge J, Dahlen G, Bergenholtz G. Dental pulp response to bacterial cell wall material. J Dent Res. (1985) 64:104650. doi: $10.1177 / 00220345850640080401$

40. Jontell M, Okiji T, Dahlgren U, Bergenholtz G. Immune defense mechanisms of the dental pulp. Crit Rev Oral Biol Med. (1998) 9:179-200 doi: 10.1177/10454411980090020301

41. Sundqvist G. Ecology, and pathogenicity of the root canal flora. Oral Surg Oral Med Oral Pathol. (1994) 78:522-30. doi: 10.1016/0030-4220(94)90047-7

42. Sundqvist G. Associations between microbial species in dental root-canal infections. Oral Microbiol Immun. (1992) 7:25762. doi: 10.1111/j.1399-302X.1992.tb00584.x

43. Sundqvist G. Ecology of the Root-Canal Flora. J Endodont. (1992) 18:42730. doi: 10.1016/S0099-2399(06)80842-3

44. Hahn CL, Liewebr FR. Relationships between caries bacteria, host responses, and clinical signs and symptoms of pulpitis. J Endodont. (2007) 33:2139. doi: 10.1016/j.joen.2006.11.008

45. Khabar KS, Al-Zoghaibi F, Al-Ahdal MN, Murayama T, Dhalla M, Mukaida $\mathrm{N}$, et al. The alpha chemokine, interleukin 8 , inhibits the antiviral action of interferon alpha. J Exp Med. (1997) 186:1077-85. doi: 10.1084/jem.186.7.1077

46. Cunha FQ, Lorenzetti BB, Poole S, Ferreira SH. IL-8 as a mediator of sympathetic pain. Br J Pharmacol. (1991) 104:7657. doi: 10.1111/j.1476-5381.1991.tb12502.x

47. Khan J, Hassun H, Zusman T, Korczeniewska O, Eliav E. Interleukin-8 levels in rat models of nerve damage and neuropathic pain. Neurosci Lett. (2017) 657:106-12. doi: 10.1016/j.neulet.2017.07.049

48. Parkitny L, McAuley JH, Di Pietro F, Stanton TR, OConnell $\mathrm{NE}$, Marinus J, et al. Inflammation in compex regional pain syndrome. A systemaic review and metanalysis. Neurology. (2013) 80:106-17. doi: 10.1212/WNL.0b013e31827blaal

49. Silva AC, Faria MR, Fontes A, Campos MS, Cavalcari BN. Interleukin-1 beta and interleukin-8 in healthy and inflamed dental pulps. J Endodontics. (2008) 17:527-32. doi: 10.1590/S1678-77572009000500031

Conflict of Interest: The authors declare that the research was conducted in the absence of any commercial or financial relationships that could be construed as a potential conflict of interest.

Copyright () 2019 Pluta, Yousefi, Damania and Khan. This is an open-access article distributed under the terms of the Creative Commons Attribution License (CC BY). The use, distribution or reproduction in other forums is permitted, provided the original author(s) and the copyright owner(s) are credited and that the original publication in this journal is cited, in accordance with accepted academic practice. No use, distribution or reproduction is permitted which does not comply with these terms. 